\title{
La producción científica sobre televisión en comunicación: un estudio sistemático en Web of Science
}

\section{Scientific production on television in communication: a systematic study via Web of Science}

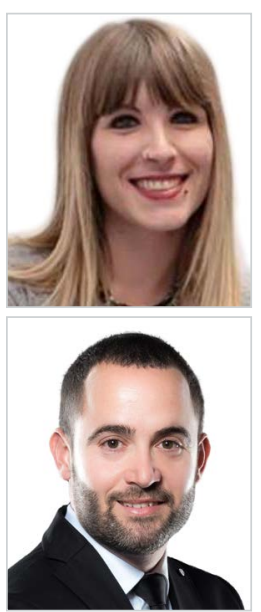

Recibido: 25/05/2021 - Aceptado: 30/09/2021 - En edición: 18/10/2021 - Publicado: 01/12/2021

\section{Resumen:}

El presente trabajo analiza la producción científica sobre televisión en el área de comunicación, con el objetivo de detectar el estado actual de la cuestión, tendencias en los temas y subtemas más investigados sobre televisión y diferencias de género en las autorías. Para ello, se realiza una búsqueda bibliográfica sistemática de los artículos publicados en las revistas científicas del área de Comunicación de la colección principal de Web of Science desde 2016 hasta 2020 en español. El análisis de la muestra, compuesta por un total de 192 artículos, permite concluir una tendencia a la baja en lo que a la investigación sobre televisión se refiere y pone de manifiesto que conceptos como la audiencia social, el audiovisual o los géneros informativos y de ficción son los más habituales en las publicaciones sobre televisión en español.

\section{Palabras clave:}

Televisión; comunicación científica; revistas; investigación; palabras clave; Web of Science; artículos; género.
Received: 25/05/2021 - Accepted: 30/09/2021 - Early access: 18/10/2021 - Published: 01/12/2021

\section{Abstract:}

This paper analyses the scientific production on television in the area of communication, with the aim of detecting the current state of the art, trends in the most researched topics and subtopics on television and gender differences in authorship. To this end, a systematic bibliographic search of articles published in scientific journals in the area of Communication in the main collection of Web of Science from 2016 to 2020 in Spanish was carried out. The analysis of the sample, composed of a total of 192 articles, allows us to conclude a downward trend in terms of research on television and shows that concepts such as social audience, audiovisual or news and fiction genres are the most common in publications on television in Spanish.

\section{Keywords:}

$T V$; scientific communication; journals; research; keywords; Web of Science; papers; gender.

Cómo citar este artículo:

Hidalgo-Marí, T. y Segarra-Saavedra, J. (2021). La producción científica sobre televisión en comunicación: un estudio sistemático en Web of Science. Doxa Comunicación, 33, pp. 363-376.

https://doi.org/10.31921/doxacom.n33a858 


\section{Introducción y estado de la cuestión}

La televisión ha sido, sin duda, el medio del siglo XX. A pesar de su tardío surgimiento y desarrollo en comparación a otros mas media como la prensa o la radio, el audiovisual doméstico ha sido considerado por la academia como el medio de medios, hasta la llegada de Internet. Su alcance doméstico y para todos los públicos lo convirtieron, desde prácticamente sus orígenes, en una ventana al mundo exterior, una metáfora sobre la que han reflexionado distintos autores y autoras.

En lo que a la producción científica se refiere, la televisión ha sido, desde prácticamente su desarrollo, uno de los temas latentes de investigación académica desde distintas perspectivas y ámbitos del conocimiento. El pensamiento de la Escuela de Frankfort aportó, sin duda, estudios preliminares que pensaban la televisión desde una perspectiva crítica dentro de la cultura de masas (Adorno y Revol, 1966). La posibilidad de proyectarse sobre el propio espectador, tal y como afirmaba McLuhan (1964), hace que el medio televisivo se convierta en un referente a investigar en las Ciencias Sociales en general y en la comunicación en particular, debido a su gran capacidad de influencia y modelación sobre las personas en las que se proyecta. Dicho de otro modo, la propias características técnicas y narrativas convierten a la televisión en un medio de especial interés sobre el que las Ciencias Sociales proyectan sus investigaciones.

Los estudios sobre televisión han evolucionado desde lo general hacia lo particular, siguiendo la tendencia general de la investigación en comunicación. Las primeras reflexiones teóricas sobre la televisión como industria cultural (Adorno y Revol, 1966; Horkheimer y Adorno, 1969), que proliferaron en las décadas de los sesenta y parte de los setenta y que han servido de fundamento teórico a las investigaciones posteriores, fueron derivando en estudios de caso cada vez más concretos, en un intento de dar respuesta a todas las bifurcaciones que el medio televisivo alberga (Bustamante, 1993).

El estudio histórico del medio televisivo supone uno de los ejes vertebrales de la producción científica sobre televisión, tal y como se demuestra en trayectorias investigadoras como la de Eco (1986; 1990), Castells (1991), Baget (1993), Ruíz del Olmo (1997), Palacio (1992) o Bustamante y Zallo (1988), pero a pesar de que el estudio histórico de la televisión supone la línea general de la investigación sobre el medio, se podría decir que es a partir de los años noventa, coincidiendo con la apertura del mercado televisivo español, el auge del desarrollo de los contenidos y la lucha por las audiencias, el momento en el que los estudios sobre televisión resurgen de forma consolidada. Así, se empiezan a publicar trabajos desde distintas perspectivas, que abordan la televisión como industria cultural reinventada y evolucionada (Bustamante, 2013; Martín-Barbero, 2015) pero también proliferan los trabajos sobre contenidos y programas, los estudios semióticos sobre el medio (Vilches, 2017) o la publicidad en televisión (Degrado, 2005; Torres y Muñoz, 2006) y la recepción de la audiencias (Morley, 1996; Quintas-Froufe y González-Neira, 2014), así como la investigación sobre los géneros televisivos (Wolf, 1984; Martín-Barbero y Muñoz, 1992; Mazziotti, 2001; Soulages, 2005; Tous, 2010), pasando por estudios sobre la convergencia del medio (Scolari, 2009; Jenkins, 2012) o su poder para influir en la construcción social de los espectadores (Galán, 2007; Lacalle e Hidalgo-Marí, 2016; Hidalgo-Marí, 2017). Por su parte, los estudios de caso y, en concreto, las investigaciones sobre la televisión local marcan una línea paralela de trabajo, como se aprecia en los trabajos de Marzal y Casero $(2008$; 2009) y en otros estudios de caso que han abordado el fenómeno desde distintas perspectivas (Prado, 2004; Román, 2005; López-Cantos, 2013). Sin embargo, en el siglo XXI han visto la luz trabajos y comprendidos que han seguido alimentando el estudio histórico e histórico reciente del medio televisivo, como se aprecia en las obras de Montero (2018) o el trabajo de Martín-Jiménez sobre la programación histórica en España (2013). 
El interés por el medio televisivo, que permite situar este asunto entre las temáticas recurrentes en los estudios sobre comunicación, se puede apreciar, también, en la cantidad de tesis doctorales defendidas en España sobre el tema. Como afirman Repiso, Torres y Delgado (2011, p. 153) en un estudio que identifica 404 tesis doctorales defendidas en España entre 1976 y 2007 :

"la evolución en el número de tesis en comparación con todas las leídas en el territorio nacional nos indica que la investigación sobre televisión ha tenido un ritmo de crecimiento y maduración similar a las tendencias globales en España. Algo que, sin duda, es un dato relevante si tenemos en cuenta el carácter reciente de los estudios sobre comunicación en las facultades españolas".

En el mismo trabajo, los autores evidencian cifras que, sin duda, sitúan a la investigación sobre temas televisivos en una buena posición en lo que a los estudios del área de comunicación se refieren, al afirmar que:

"El número total de tesis doctorales defendidas sobre televisión en España entre 1976/2007 ha sido de 404 (...) constatándose un crecimiento continuo en concordancia con el resto de la producción española. La producción de tesis sobre televisión comenzó a finales de las década de los setenta consolidándose en los años 80. Sin embargo, no es hasta finales de los noventa cuando se produce un despegue significativo con las 129 tesis doctorales leídas en el quinquenio 1998/2002. El mayor nivel de producción se alcanza en el último período (2003/07) con 142 tesis, aunque la producción ya crece de forma menor y parece estabilizada en torno a esas cifras" (Repiso, Torres y Delgado, 2011, p. 154).

Si se tiene en cuenta que la producción de tesis doctorales es el primer paso para futuras publicaciones científicas en revistas, se puede apreciar que el interés por el medio televisivo en la academia ha sido constante desde la consolidación del medio, aunque resulta evidente que el crecimiento en la producción científica, según los datos aportados por los autores citados, se concentra en el momento en el que se desarrollan las televisiones privadas en España, coincidiendo con la década de los noventa y que se mantiene constante a medida que avanza el desarrollo del medio. Aunque se augura cierta relación entre los avances del medio televisivo y el aumento de la producción científica al respecto, otros trabajos previos sobre la producción televisiva indican que no es posible establecer una relación directa entre los hitos experimentados por el medio televisivo y la producción científica al respecto, como afirman Castillo y Soler (2014, s.p.) en su trabajo sobre la investigación en documentación televisiva:

"No podemos relacionar acontecimientos con un aumento del número de publicaciones, como por ejemplo la aparición de televisiones autonómicas o privadas, pero sí se ve un incremento en lo relacionado con la digitalización. Se habla más de ella en 1986 y en 2007. Este último año coincidente con las fechas en que se produjo el cambio de lo analógico a lo digital”.

No podemos establecer una correlación entre la temática de las publicaciones y los acontecimientos producidos en las fechas de dichas publicaciones, sino que la mayor o menor edición de artículos o comunicaciones a congresos centrados en la documentación televisiva suele corresponder con números especiales dedicados a ella o con la salida de números de revistas especializadas en documentación de medios.

Algunos estudios han profundizado en el estado de la investigación científica sobre temas específicos, como se aprecia en el trabajo de Ortíz, Ruíz y Díaz (2013, p. 138), que trata la investigación sobre televisión e infancia, destacando algunos proyectos específicos que han desarrollado en profundidad el tema: 
"El estudio de la relación de los nativos digitales con las pantallas (García, 2009: 7- 30) a través del Proyecto Socmedia, o iniciativas para el estudio de la relación entre menores y tele vi - sión -como el Proyecto Procotin (2008-12), dirigido por Núñez-Ladeveze son solo algunas de las iniciativas que, desde hace años, vienen sucediéndose en España".

Más recientemente, y con la llegada de las plataformas digitales y la televisión on demand a España, el papel de la televisión ha sido cuestionado y reflexionado por la academia con más viveza que nunca. Así, mientras las redes sociales e Internet aparecen ser las temáticas preferidas entre los grupos de investigación, lo cierto es que la televisión, fruto del nuevo impacto tecnológico y del avance del desarrollo tecnológico, sigue suscitando un interés notable entre la comunidad investigadora.

Por todo ello, el presente trabajo tiene como objetivo principal realizar una revisión sistemática de las publicaciones españolas sobre televisión en el área de comunicación con el fin de detectar el estado de la cuestión de la misma y, a su vez, poder detectar temáticas y tendencias, así como corrientes más prolíficas para ser publicadas. Mediante un estudio de los artículos publicados en Web of Science (WOS) y el análisis de sus keywords, se pretende detectar qué temáticas y subtemas están generando un mayor interés por la comunidad investigadora, siempre dentro del prisma televisivo.

\section{Objetivos y metodología}

El objetivo general del presente trabajo reside en abordar el fenómeno de la producción científica sobre televisión en el área de comunicación. Como objetivos específicos, se plantean los siguientes:

- (OE1) Conocer la evolución de los estudios sobre televisión publicados en la WOS en los últimos cinco años (2016-2020).

- (OE2) Identificar y analizar tendencias en las subtemáticas y géneros predominantes en la producción científica sobre televisión en WOS.

- (OE3) Detectar diferencias entre la presencia y liderazgo de hombres y mujeres frente a la investigación en televisión.

Para poder alcanzar los objetivos expuestos se ha trabajado con una metodología cuantitativa basada en la búsqueda bibliográfica sistemática y su posterior clasificación. La Web os Science, según se define en su propia web, es un servicio digital de documentación científica, administrado por Clarivate Analytics que se encuentra integrado en el ISI Web of Knowledge (WoK) que permite el acceso a una amplia colección de bases de datos en las que se recogen artículos y citas de revistas científicas, libros y otros tipos de material científico de todos los campos del conocimiento académico y que, además, establece criterios de ordenación sobre la calidad de la publicación científica.

Dicha búsqueda sistemática se ha llevado a cabo en la base de datos de la Web of Science, utilizando como concepto genérico “televisión". Una vez identificados y listados todos los documentos indexados en cuyo título aparecía la palabra clave "televisión”, dentro de la colección principal de Web of Science, se han seleccionado solamente aquellos artículos cuya lengua original es el español y se han excluido libros, capítulos de libro, reviews y otros formatos. La búsqueda se ha acotado a una ventada de cinco años (2016-2020) por considerar que el 2016 fue un año clave en la transformación de la televisión, con el aterrizaje en España de las grandes plataformas de contenido bajo demanda (VOD), algo que, sin duda, marcó un antes y un después en la historia reciente de la televisión. Partimos de la hipótesis de que los estudios de televisión se multiplicaron considerablemente debido 
al fenómeno productivo y que el interés de la comunidad investigadora en comunicación sobre el medio televisivo y sus nuevas formas de producción y consumo se han visto plasmados en la producción académica y científica de los últimos años.

Tras aplicar los criterios indicados, WOS devuelve 195 artículos científicos que se someten a revisión por parte del equipo investigador, con el fin de detectar posibles incoherencias en la búsqueda. Esta acción manual detecta tres trabajos que pertenecen a libros colectivos y que se encuentran etiquetados como artículos y son eliminados por el equipo. La muestra final sobre la que se trabaja en este manuscrito es de 192 artículos científicos en español publicados en revistas de la Web of Science (WOS) del área de comunicación desde 2016 hasta 2020.

Obtenida la muestra, se ha procedido al análisis y clasificación de la información, así como a la aplicación de los cálculos estadísticos necesarios. Para poder establecer tendencias temáticas, se ha tenido como referencia el uso de las keywords propuestas por los autores. Dichas palabras clave han sido sometidas a clasificación individual y puestas en común, con el fin de detectar tendencias sobre conceptos y tags más utilizados.

\section{Resultados}

La muestra sobre la que se trabaja se compone de 192 artículos científicos publicados en español desde 2016 hasta 2020 . Si se analiza la publicación anual de artículos sobre televisión se detecta una tendencia evidente a la baja (ver gráfico 1), en lo que respecta al año de inicio del estudio, que se encuentra en el punto más alto de la publicación con 55 artículos recogidos, lo que supone un $\mathbf{2 8 . 5 \%}$ del total de los artículos publicados en el lustro analizado.

Una caída del 60\% en la producción desde el año 2016 hasta el 2017 indica un descenso considerable en los trabajos sobre televisión, que cambia de tendencia en el 2018, que permite vislumbrar cierto resurgir en lo que a la publicación sobre televisión se refiere.

No obstante, este aumento puntual de artículos publicados supone el punto de inflexión a partir del cual la publicación sobre televisión decae en los años posteriores, hasta 29 trabajos en el año 2020, lo que supone, prácticamente, la mitad de los artículos científicos sobre televisión que en el año de inicio de esta investigación. 


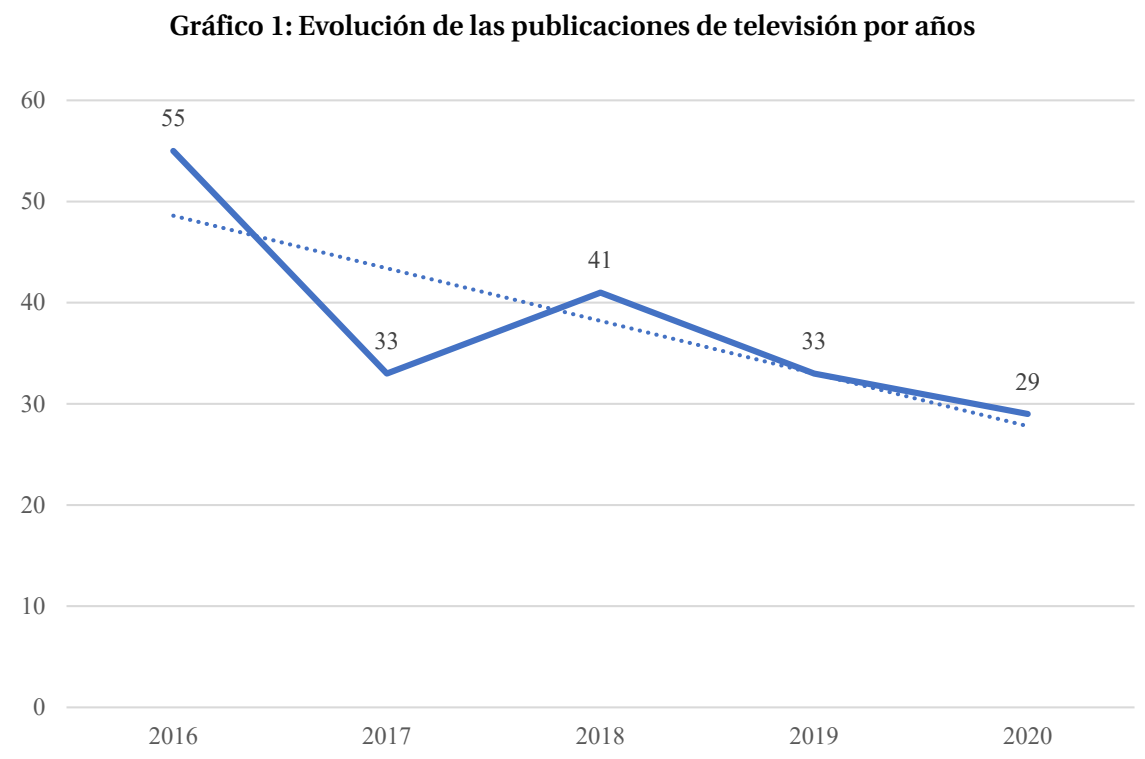

Fuente: elaboración propia

\subsection{Sobre las publicaciones}

Los 192 artículos se publican en 33 revistas distintas, de las cuales, 23 (69,7\%) son españolas, 9 (27,3\%) iberoamericanas y 1 (3\%) portuguesa, lo que indica a primera vista que las revistas españolas de comunicación son propicias a la publicación de textos que traten la comunicación en televisión.

Dentro de las revistas iberoamericanas, son las revistas chilenas las que presentan una mayor presencia (un 9\% del total, es decir, 3 revistas), siendo estas Cuadernos.info, Comunicación y medios y Perspectivas de la comunicación. Argentina alberga dos revistas de la muestra y el resto de los países presentes (Uruguay, Perú, Colombia y Ecuador) solo registran una revista, al igual que Portugal (ver gráfico 2).

Ahora bien, a pesar de que el volumen de revistas españolas supera significativamente al resto de revistas, si se hace un análisis que relacione el número de publicaciones sobre televisión y la procedencia de las revistas, se observa que el 28,64\% de los artículos de la muestra están publicados por revistas iberoamericanas, algo que lleva a pensar que a pesar de que hay más revistas españolas que publican textos sobre televisión, el volumen de artículos publicados por las revistas iberoamericanas es relevante. La longevidad de algunas revistas iberoamericanas podría justificar este dato puesto que algunas de ellas como Revista Chasqui (que publica ininterrumpidamente desde 1981) o Palabra clave (publicando desde 1996) presentan una trayectoria temporal mucho más amplia que las revistas españolas. 
Gráfico 2: Distribución de revistas científicas por países

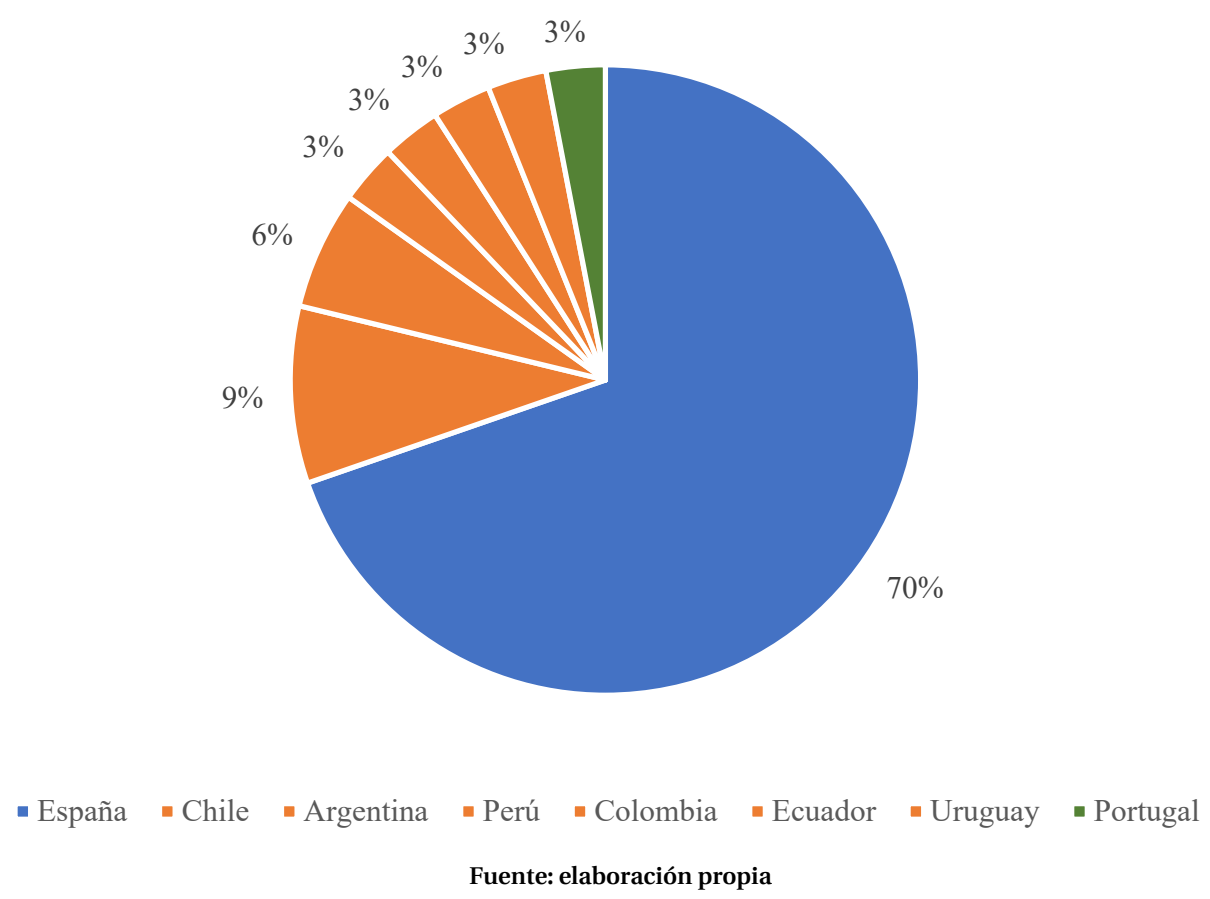

Tal y como se puede ver en el gráfico 3, la revista con más artículos sobre televisión publicados es Revista Latina de Comunicación Social, que alberga el 17,27\% del total de artículos, seguida de Profesional de la información, con un 12\% y la revista Index.Comunicación, con un 7,85\% del total de artículos. Se han excluido del gráfico las revistas que presentan menos de cuatro artículos publicados. 
Gráfico 3: Distribución de artículos por revistas científicas

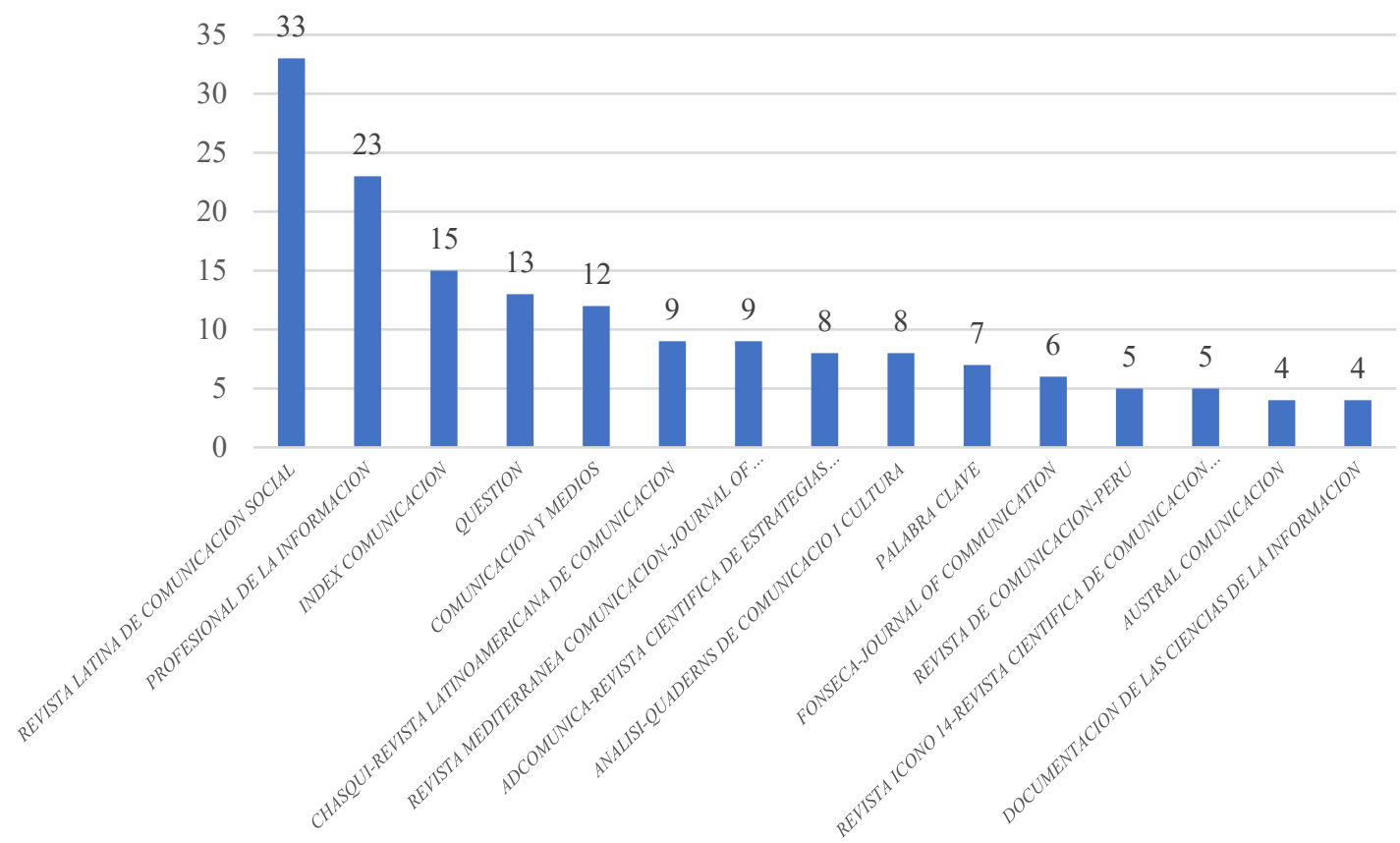

Fuente: elaboración propia

\subsection{Temas y subtemas en la investigación sobre televisión (keywords)}

Si se analizan las palabras clave de los 192 artículos sobre televisión, se detecta que el concepto más repetido, obviamente, es el de televisión, aunque otros conceptos parecen mostrar cierta tendencia en los estudios sobre este medio. El término "televisión" aparece en el $91 \%$ de los artículos analizados, junto al resto de conceptos (ver gráfico 4). Resulta evidente esta presencia significativa si tenemos en cuenta que estamos haciendo alusión al concepto que ha servido de filtrado inicial para la obtención de la muestra, por lo que merece la pena desglosar de forma más detallada el resto de conceptos que permiten codificar temas y subtemas presentes en la producción científica seleccionada. 


\section{Gráfico 4: Top de keywords más frecuentes en \%}

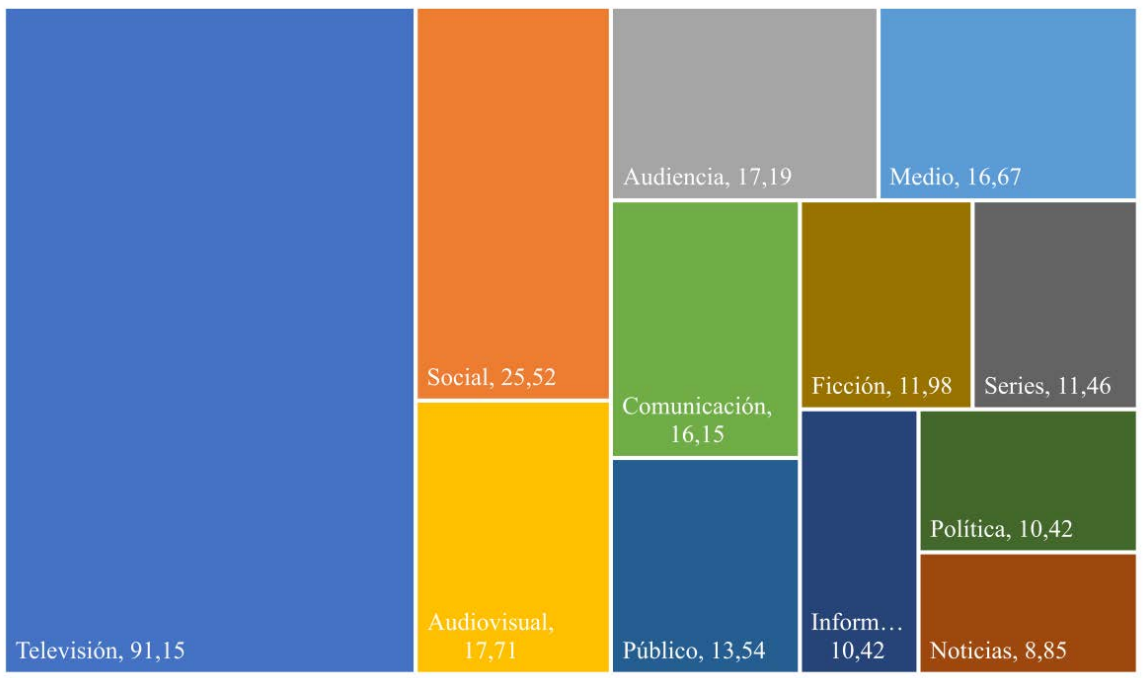

Fuente: elaboración propia

El segundo término con más frecuencia es el tag "social”, que aparece en un $25.52 \%$ de los artículos analizados, seguido del concepto "audiovisual" que aparece en el $17.70 \%$ de los casos. La presencia relevante de la palabra "social "nos indica cómo la transformación del medio televisivo hacia una televisión más interactiva, más personalizada y, por tanto, más social, es un tema recurrente que genera interés entre la comunidad investigadora, aunque no se puede obviar la vinculación existente con temáticas sociales, que podría explicar el fenómeno. El concepto "audiencia” también ocupa un lugar prioritario en las investigaciones sobre televisión, apareciendo en un 17,18\% de los artículos, algo que evidencia que los estudios sobre la recepción del medio, tan relevantes en décadas pasadas, siguen teniendo incidencia en la investigación sobre televisión reciente. Las redes sociales, por su parte, solo aparecen en un $8,3 \%$ de los artículos analizados y normalmente suele utilizarse este concepto acompañado del término "audiencia". Resulta curioso cómo una temática tan patente en los tiempos que corren como son los social media, no adquieren un protagonismo significativo en los estudios sobre televisión.

Más relevante, en términos cualitativos, resulta la relevancia del concepto "audiovisual" (presente en un 17\% de los artículos analizados) que, si bien hace alusión a una característica técnica del medio televisivo, al ser usado como keyword aborda un escenario más propio del estudio narrativo de la televisión y sus discursos, que del propio elemento técnico.

Los conceptos “comunicación” y “medio”, permiten indexar los artículos no solo por temática, sino también dentro de un área de conocimiento determinado. Sin embargo, a pesar de que adquieren una presencia a destacar en la totalidad de la muestra, ambos conceptos están presentes en un 16\% del total de artículos analizados. 
El concepto “información" en los estudios sobre televisión aparece en un 10,41\% de la muestra, y en concreto, el concepto "noticia" se utiliza en un $\mathbf{8 . 8 5 \%}$ del total de la muestra. Si se unen los dos conceptos clave que pueden representar el estudio de noticias e información de actualidad, podemos acercarnos casi al 20\%, aunque la dispersión en los términos de indexación no permitiría afirmar una presencia mayor que la de otros conceptos mencionados.

Mención aparte merece la indexación de los géneros de estudio. Alrededor de un $12 \%$ de los artículos se centran en el estudio de la ficción televisiva (sin especificar formatos) y un $8 \%$ del total lo hacen, en concreto, bajo el concepto de "series" (11.45\%), mientras que otros formatos televisivos como la telerrealidad o el espectáculo en directo (bajo el tag show) a penas representan un $2.6 \%$ y un $1.5 \%$ del total de la muestra, respectivamente.

Otro concepto que ha devuelto un porcentaje a comentar es el concepto "público". Tanto vinculado a las cadenas públicas como mediante su relación con la convergencia pública, aparece en alrededor del 13\% de los artículos analizado, seguido del concepto "política" que aparece en un 10,41\%, algo que evidencia la importancia para la comunidad investigadora de la comunicación política derivada del medio televisivo.

Imagen 1: Nube de palabras más utilizadas

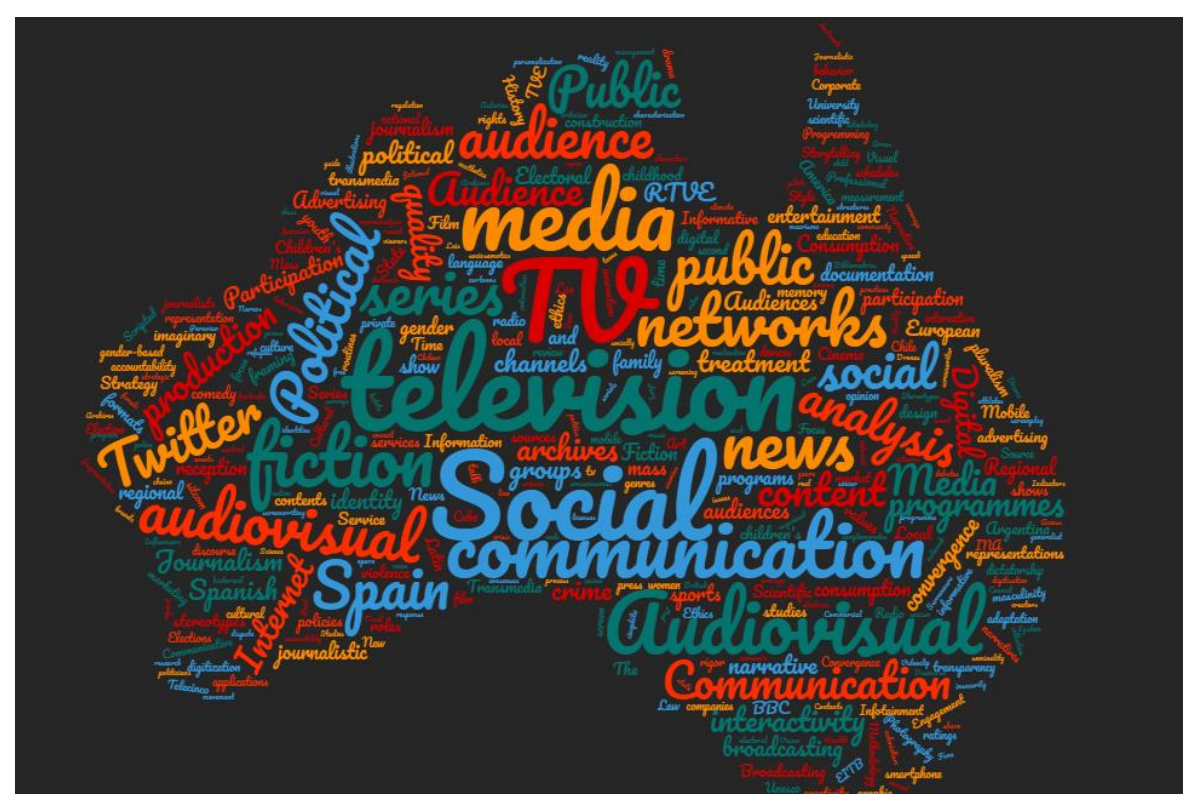

Fuente: elaboración propia a partir de nubedepalabras.com 
En un segundo nivel de aparición se encuentran conceptos que, a pesar de su relevancia, no aparecen con una frecuencia relevante en los artículos sobre televisión. Nos referimos, por ejemplo, a la palabra "Internet”, que solo aparece en un $4.61 \%$ del total de artículos u otras como "programas", "digital", "interactividad” o "canales" que apenas superan una presencia del 4\%. Al aplicar un estudio pormenorizado de las palabras clave que tienen una presencia en los artículos sobre televisión inferior al $4 \%$, se detectan aspectos a destacar, como la escasa incidencia de artículos que utilicen el concepto "convergencia" (2,60\%) a pesar de la actualidad del término u otros con una presencia similar como "publicidad" (3,60\%), "programas" o "entretenimiento", ambas con una presencia del 2,08\%. Tampoco tienen una presencia significativa los estudios de género aplicados al medio televisivo, pues solo se encuentran en un $2 \%$ de los trabajos analizados.

\subsection{Autorías y género}

Los 192 artículos analizados están firmados por un total de 398 personas autoras de distintas procedencias y áreas, de las cuales, un $55,41 \%$ son mujeres y un 44,59\% son hombres. A pesar de que la diferencia no es significativa, se detecta un mayor interés por el fenómeno televisivo por parte de las investigadoras mujeres.

Otro dato relevante a tener en cuenta es que dada la participación de diversas personas en el proceso de investigación, redacción y publicación de los artículos, se debe conocer el grado de participación colaborativa en las investigaciones. En este sentido, se detecta que el promedio de coautoría por artículo se sitúa en 2,1.

Las mujeres son las primeras firmantes del $60 \%$ de los trabajos publicados y en el $37 \%$ de los casos aparecen como segundas autoras, algo que permite vislumbrar cierto liderazgo en lo que a investigaciones sobre televisión se refiere. Además, solo en el $26 \%$ de los trabajos no aparece ninguna mujer firmante, lo que confirma la implicación del género femenino en la investigación sobre televisión.

\section{Conclusiones}

La exposición cuantitativa previa permite afirmar, en primer lugar, que existe una tendencia a la baja en lo que a la publicación de artículos sobre televisión en español se refiere. Se partía de la hipótesis, al iniciar este trabajo, que el auge que vive la televisión digital en la actualidad sería un sinónimo de un mayor interés por la investigación académica, algo que se refuta después de la exposición de resultados.

Una reflexión más cualitativa al respecto lleva a pensar que el motivo pueda derivar de la búsqueda de la internacionalización de las personas autoras y las exigencias del sistema por la publicación en lenguas distintas al español (en inglés, fundamentalmente), si bien la información que devuelve esta investigación no permite más que reformular una nueva hipótesis al respecto.

El hecho de que la publicación de textos en español sobre televisión haya caído significativamente (recordemos, más del 50\% menos desde el 2016 hasta el 2020) resulta cuanto menos, preocupante, si se tienen en cuenta los cambios que el medio televisivo está experimentando y, con ello, los cambios en la forma de consumir, de producir y de crear industria alrededor de la televisión y las plataformas digitales. 
En lo que a las tendencias y subtendencias de investigación se refiere, se puede afirmar que los estudios que mayor interés suscitan por parte de la comunidad investigadora y por parte de las revistas que publican está concentrada, en su gran mayoría en los estudios sobre televisión social y audiencias, dos conceptos que, si se unen, permiten detectar la importancia que desde la academia se está dando a las nuevas formas de recepción y consumo audiovisual.

Los estudios específicos sobre géneros televisivos parecen focalizarse en la información y dentro de ésta, en los noticiarios y tratamiento de noticias, poniendo de manifiesto la importancia del género periodístico dentro del medio televisivo. De la mano de la información, destaca la investigación sobre productos de ficción y, concretamente, sobre series de televisión, que se postulan como los formatos más fecundos en la investigación sobre televisión, dejando de lado el cine, los documentales o los programas de entretenimiento. El auge en el interés de la ficción televisiva parece coincidir con el boom de la producción de ficción seriada tanto en España como en el resto de los países, que está copando la oferta de contenidos tanto en la televisión convencional como en las plataformas digitales.

En lo que a la representación de la autoría por géneros se refiere, se ha detectado que existe un compromiso real por la comunidad femenina académica en la investigación sobre televisión, a pesar de que el porcentaje de mujeres es ligeramente superior, por lo que no se puede descartar el interés por parte de la comunidad masculina en la investigación sobre televisión.

Se concluye, además, que los datos analizados indican que los estudios sobre televisión en español han descendido considerablemente en los últimos cinco años, si bien es cierto que este descenso podría explicarse por el trance que experimenta el medio televisivo. A pesar de las evidencias de este trabajo, se detecta la necesidad de ampliar el estudio en lo que al periodo temporal se refiere, con el fin de poder responder a nuevas hipótesis que confirmen una tendencia decadente en la investigación sobre televisión en el área de comunicación, así como una comparativa entre disciplinas afines que permitiera valorar si las investigaciones sobre televisión han migrado a otros campos o, por el contrario, la tendencia a la baja en dicha temática es común al resto de áreas.

\section{Agradecimientos}

Este artículo ha sido traducido por Lucía Otaola Puche.

Artículo realizado en el marco del proyecto de investigación emergente del Vicerrectorado de Investigación y Transferencia de Conocimiento de la Universidad de Alicante, "Ficción online a la carta: Producción, contenido e interacción en las series españolas de las plataformas de TV in streaming (2016-2019)" (GRE 19-10) y en el proyecto de investigación “Nuevos valores, gobernanza, financiación y servicios audiovisuales públicos para la sociedad de Internet: contrastes europeos y españoles (VALPSM2020)" (RTI2018-096065-B-I00).

\section{Referencias bibliográficas}

Adorno, T. W. y Revol, E. L. (1966). Televisión y cultura de masas. Eudecor.

Baget, J. M. (1993). Historia de la televisión en España, 1956-1975. Feedback Ediciones.

Bustamante, E. (1993). La Economía de la televisión. Gedisa.

374 | n³3, pp. 363-376 | doxa.comunicación

julio-diciembre de 2021 
Bustamante, E. (2013). Historia de la radio y la televisión en España. Gedisa.

Bustamante, E. y Zallo, R. (Coords.) (1988). Las industrias culturales en España. Aka.

Castells, M. (1991). La era de la información: Economía, sociedad y cultura. Alianza.

Castillo Blasco, L. \& Soler Monreal, C. (2014). Análisis de los artículos publicados sobre documentación televisiva en España (1984-2014). BiD: textos universitaris de biblioteconomia i documentación, 33, s.p. http://dx.doi.org/10.1344/BiD2014.33.5

Degrado-Godoy, M. D. (2005). Televisión, publicidad y comunicación. Comunicar: Revista Científica de Comunicación y Educación, 13(25). https://doi.org/10.3916/C25-2005-078

Eco, U. (1986). TV: la transparencia perdida. En Eco, U., La estrategia de la ilusión, 200-223. DeBolsillo.

Eco, U. (1990). A Guide to the Neo-Television of the 1980s. In Culture and conflict in postwar Italy (pp. 245-255). Palgrave Macmillan.

Galán, E. (2007). Construcción de género y ficción televisiva en España. Revista Comunicar, 14(28), 229-236. https://bit.ly/3feHnGj Hidalgo-Marí, T. (2017). De la maternidad al empoderamiento: una panorámica sobre la representación de la mujer en la ficción española. Prisma Social, (2), 291-314. https://bit.ly/34eSCs0

Horkheimer, M. y Adorno, T. (1969). La industria cultural. Industria cultural y sociedad de masas. Monte Ávila.

Jenkins, H. (2012). Textual poachers: Television fans and participatory culture. Routledge.

Lacalle, C. \& Hidalgo-Marí, T. (2016). La evolución de la familia en la ficción televisiva española. Revista Latina de Comunicación Social, (71), 470-483. https://doi.org/10.4185/RLCS-2016-1105

López Cantos, F. J. (2003). La televisión local en el contexto audiovisual. Análisis del sector en la provincia de Castellón (Tesis Doctoral, Universitat Jaume I).

Martín-Barbero, J. (2015). La Televisión: una cuestión de espacio. En Pereira, J.M. (Ed.), Televisión y construcción de lo público, 61-70.

Martín-Barbero, J. y Muñoz, S. (1992). Televisión y melodrama. Tercer Mundo.

Martín-Jiménez, V. (2013). Televisión Española y la Transición democrática: la comunicación política del cambio (1976-1979). Valladolid: Universidad de Valladolid.

Marzal Felici, J. \& Casero-Ripollés, A. (2008). La investigación sobre la televisión local en España: nuevas agendas ante el reto de la digitalización. Facultad de Ciencias Sociales y de la Comunicación, Universidad del País Vasco.

Marzal Felici, J. \& Casero-Ripolles, A. (2009). Las políticas de comunicación ante la implantación de la TDT en España. Balance crítico y retos pendientes. Universidad Católica San Antonio de Murcia. Facultad de Ciencias Sociales y de la Comunicación.

McLuhan, M. (1964). Understanding media. McGraw-Hill.

Mazziotti, N. (2001). Los géneros en la televisión pública. En Rincón, O. (Comp.). Televisión Pública: del consumidor al ciudadano. Convenio Andrés bello.

Montero, J. (2018). Una televisión con dos cadenas la programación en España (1956-1990). Madrid: Cátedra. 
Morley, D. (1996). Interpretar televisión: la audiencia de Nationwide. En Moreley, D. Televisión, audiencias y estudios culturales. Amorrortu.

Palacio, M. (1992). Una historia de la televisión en España: Arqueología y vanguardia. Madrid Capital Europea de la Cultura. Prado, E. (2004). La televisión local entre el limbo regulatorio y la esperanza digital. Fundación Alternativas.

Ortíz-Sobrino, M.A., Ruíz-San-Román, J.A. y Díaz-Cerveró, E. (2013). ¿Están las cadenas de televisión interesadas en una mejor TV para los menores? Las televisiones y la investigación en infancia y televisión. Revista Comunicar, 20(40), 137-144. https://doi. org/10.3916/C40-2013-03-04

Quintas-Froufe, N., y González-Neira, A. (2014). Audiencias activas: participación de la audiencia social en la televisión. Comunicar: Revista Científica de Comunicación y Educación, (43), 83-90. https://doi.org/10.3916/C43-2014-08

Repiso, R., Torres, D., y Delgado, E. (2011). Análisis bibliométrico y de redes sociales en tesis doctorales españolas sobre televisión (1976/2007). Comunicar: Revista científica iberoamericana de comunicación y educación, (37), 151-159. https://doi.org/10.3916/ C37-2011-03-07

Román Portas, M. (2005). La televisión local en España. Sphera Publica, (5), 229-239. https://bit.ly/3fieity Ruiz del Olmo, J. (1997). Orígenes de la televisión en España. Universidad de Málaga.

Scolari, C. A. (2009). Alrededor de la(s) convergencia(s): Conversaciones teóricas, divergencias conceptuales y transformaciones en el ecosistema de medios. Signo y pensamiento, 54, 44-55. https://bit.ly/3fLjCoz

Soulages, J. C. (2005). Formato, estilo y géneros televisivos. DeSignis, (7-8), 0067-78. https://bit.ly/3wwtv08

Torres Moraga, E., y Muñoz Navarro, C. (2006). Estrategias de posicionamiento basadas en la cultura del consumidor: un análisis de la publicidad en televisión. Estudios Gerenciales, 22(100), 71-81. https://bit.ly/2SiPAjU

Tous, A. (2010). La era del drama en televisión. Perdidos, CSI, Mujeres desesperadas y House. Editorial UOC.

Vilches, L. (2017). Diccionario de semiótica y narrativas de cine y televisión. Significação: Revista de Cultura Audiovisual, 44(48), 15-31. https://doi.org/10.11606/issn.2316-7114.sig.2017.135194

Wolf, M. (1984). Géneros y televisión. Anàlisi: Quaderns de comunicació i cultura, 9, 189-198. https://bit.ly/3yvm2Al 